\title{
SCHWARZ METHODS: TO SYMMETRIZE OR NOT TO SYMMETRIZE*
}

\author{
MICHAEL HOLST $^{\dagger}$ AND STEFAN VANDEWALLE ${ }^{\dagger}$
}

\begin{abstract}
A preconditioning theory is presented which establishes sufficient conditions for multiplicative and additive Schwarz algorithms to yield self-adjoint positive definite preconditioners. It allows for the analysis and use of nonvariational and nonconvergent linear methods as preconditioners for conjugate gradient methods, and it is applied to domain decomposition and multigrid. It is illustrated why symmetrizing may be a bad idea for linear methods. It is conjectured that enforcing minimal symmetry achieves the best results when combined with conjugate gradient acceleration. Also, it is shown that the absence of symmetry in the linear preconditioner is advantageous when the linear method is accelerated by using the Bi-CGstab method. Numerical examples are presented for two test problems which illustrate the theory and conjectures.
\end{abstract}

Key words. multigrid, domain decomposition, Krylov methods, Schwarz methods, conjugate gradients, Bi-CGstab

AMS subject classifications. $65 \mathrm{~N} 55,65 \mathrm{~N} 22,65 \mathrm{~F} 10,65 \mathrm{~J} 10$

\section{PII. S0036142994275743}

1. Introduction. Domain decomposition (DD) and multigrid (MG) methods have been studied extensively in recent years, both from a theoretical and numerical point of view. DD methods were first proposed in 1869 by H. A. Schwarz as a theoretical tool in the study of elliptic problems on nonrectangular domains [22]. More recently, DD methods have been reexamined for use as practical computational tools in the (parallel) solution of general elliptic equations on complex domains [16]. MG methods were discovered much more recently [10]. They have been extensively developed both theoretically and practically since the late seventies $[6,11]$, and they have proven to be extremely efficient for solving very broad classes of partial differential equations. Recent insights into the product nature of certain MG methods have led to a unified theory of MG and DD methods, collectively referred to as Schwarz methods $[5,9,27]$.

In this paper, we consider additive and multiplicative Schwarz methods and their acceleration with Krylov methods for the numerical solution of self-adjoint positive definite (SPD) operator equations arising from the discretization of elliptic partial differential equations. The standard theory of conjugate gradient acceleration of linear methods requires that a certain operator associated with the linear method - the preconditioner-be symmetric and positive definite. Often, however, as in the case of Schwarz-based preconditioners, the preconditioner is known only implicitly, and symmetry and positive definiteness are not easily verified. Here, we try to construct natural sets of sufficient conditions that are easily verified and do not require the explicit formulation of the preconditioner. More precisely, we derive conditions for the constituent components of MG and DD algorithms (smoother, subdomain solver, transfer operators, etc.), that guarantee symmetry and positive definiteness of the preconditioning operator which is (explicitly or implicitly) defined by the resulting Schwarz method.

${ }^{*}$ Received by the editors October 17, 1994; accepted for publication (in revised form) June 20, 1995. This research was supported in part by NSF cooperative agreement CCR-9120008.

http://www.siam.org/journals/sinum/34-2/27574.html

$\dagger$ Applied Mathematics, California Institute of Technology, Mail Code 217-50, Pasadena, CA 91125 (holst@ama.caltech.edu, stefan@ama.caltech.edu). 
We examine the implications of these conditions for various formulations of the standard DD and MG algorithms. The theory we develop helps to explain the often observed behavior of a poor or even divergent MG or DD method which becomes an excellent preconditioner when accelerated by a conjugate gradient method. We also investigate the role of symmetry in linear methods and preconditioners. Both analysis and numerical evidence suggest that linear methods should not be symmetrized when used alone, and only minimally symmetrized when accelerated by conjugate gradients, in order to achieve the best possible convergence results. In fact, the best results are often obtained when a very nonsymmetric linear iteration is used in combination with a nonsymmetric system solver such as Bi-CGstab, even though the original problem is SPD.

The outline of the paper is as follows. We begin in section 2 by reviewing basic linear methods for SPD linear operator equations and examining Krylov acceleration strategies. In sections 3 and 4, we analyze multiplicative and additive Schwarz preconditioners. We develop a theory that establishes sufficient conditions for the multiplicative and additive algorithms to yield SPD preconditioners. This theory is used to establish sufficient conditions for multiplicative and additive DD and MG methods and allows for analysis of nonvariational and even nonconvergent linear methods as preconditioners. A simple lemma, given in section 5, illustrates why symmetrizing may be a bad idea for linear methods. In section 6 , results of numerical experiments obtained with finite-element-based DD and MG methods applied to some nontrivial test problems are reported.

2. Krylov acceleration of linear iterative methods. In this section, we review some background material on self-adjoint linear operators, linear methods, and conjugate gradient acceleration. More thorough reviews can be found in $[12,18]$.

2.1. Background material, terminology, and notation. Let $\mathcal{H}$ be a real finite-dimensional Hilbert space equipped with the inner-product $(\cdot, \cdot)$ inducing the norm $\|\cdot\|=(\cdot, \cdot)^{1 / 2}$. $\mathcal{H}$ can be thought of as, for example, the Euclidean space $\mathbb{R}^{n}$, or as an appropriate finite element space.

The adjoint of a linear operator $A \in \mathbf{L}(\mathcal{H}, \mathcal{H})$ with respect to $(\cdot, \cdot)$ is the unique operator $A^{T}$ satisfying $(A u, v)=\left(u, A^{T} v\right) \forall u, v \in \mathcal{H}$. An operator $A$ is called selfadjoint or symmetric if $A=A^{T}$; a self-adjoint operator $A$ is called positive definite or simply positive if $(A u, u)>0 \forall u \in \mathcal{H}, u \neq 0$. If $A$ is SPD with respect to $(\cdot, \cdot)$, then the bilinear form $(A u, v)$ defines another inner-product on $\mathcal{H}$, which we denote as $(\cdot, \cdot)_{A}$. It induces the norm $\|\cdot\|_{A}=(\cdot, \cdot)_{A}^{1 / 2}$.

The adjoint of an operator $M \in \mathbf{L}(\mathcal{H}, \mathcal{H})$ with respect to $(\cdot, \cdot)_{A}$, the $A$-adjoint, is the unique operator $M^{*}$ satisfying $(M u, v)_{A}=\left(u, M^{*} v\right)_{A} \forall u, v \in \mathcal{H}$. From this definition it follows that

$$
M^{*}=A^{-1} M^{T} A .
$$

$M$ is called $A$-self-adjoint if $M=M^{*}$ and $A$-positive if $(M u, u)_{A}>0 \forall u \in \mathcal{H}, u \neq 0$.

If $N \in \mathbf{L}\left(\mathcal{H}_{1}, \mathcal{H}_{2}\right)$, then the adjoint of $N$, denoted as $N^{T} \in \mathbf{L}\left(\mathcal{H}_{2}, \mathcal{H}_{1}\right)$, is defined as the unique operator relating the inner-products in $\mathcal{H}_{1}$ and $\mathcal{H}_{2}$ as follows:

$$
(N u, v)_{\mathcal{H}_{2}}=\left(u, N^{T} v\right)_{\mathcal{H}_{1}} \quad \forall u \in \mathcal{H}_{1}, \quad \forall v \in \mathcal{H}_{2} .
$$

Since it is usually clear from the arguments which inner-product is involved, we shall often drop the subscripts on inner-products (and norms) throughout the paper, except when necessary to avoid confusion. 
We denote the spectrum of an operator $M$ as $\sigma(M)$. The spectral theory for self-adjoint linear operators states that the eigenvalues of the self-adjoint operator $M$ are real and lie in the closed interval $\left[\lambda_{\min }(M), \lambda_{\max }(M)\right]$ defined by the Rayleigh quotients

$$
\lambda_{\min }(M)=\min _{u \neq 0} \frac{(M u, u)}{(u, u)}, \quad \lambda_{\max }(M)=\max _{u \neq 0} \frac{(M u, u)}{(u, u)} .
$$

Similarly, if an operator $M$ is $A$-self-adjoint, then its eigenvalues are real and lie in the interval defined by the Rayleigh quotients generated by the $A$-inner-product. A wellknown property is that if $M$ is self-adjoint, then the spectral radius of $M$, denoted as $\rho(M)$, satisfies $\rho(M)=\|M\|$. This property can also be shown to hold in the $A$-norm for $A$-self-adjoint operators (or, more generally, for $A$-normal operators [1]).

LEMMA 2.1. If $A$ is $S P D$ and $M$ is $A$-self-adjoint, then $\rho(M)=\|M\|_{A}$.

2.2. Linear methods. Given the equation $A u=f$, where $A \in \mathbf{L}(\mathcal{H}, \mathcal{H})$ is $\mathrm{SPD}$, consider the preconditioned equation $B A u=B f$, with $B \in \mathbf{L}(\mathcal{H}, \mathcal{H})$. The operator $B$, the preconditioner, is usually chosen so that a Krylov or Richardson method applied to the preconditioned system has some desired convergence properties. A simple linear iterative method employing the operator $B$ takes the form

$$
u^{n+1}=u^{n}-B A u^{n}+B f=(I-B A) u^{n}+B f,
$$

where the convergence behavior of (4) is determined by the properties of the so-called error propagation operator

$$
E=I-B A \text {. }
$$

The spectral radius of the error propagator $E$ is called the convergence factor for the linear method, whereas the norm is referred to as the contraction number. We recall two well-known lemmas; see, for example, [17] or [20].

LEMMA 2.2. For arbitrary $f$ and $u^{0}$, the condition $\rho(E)<1$ is necessary and sufficient for convergence of the linear method (4).

LEMMA 2.3. The condition $\|E\|<1$, or the condition $\|E\|_{A}<1$, is sufficient for convergence of the linear method (4).

We now state a series of simple lemmas that we shall use repeatedly in the following sections. Their short proofs are added for the reader's convenience.

LEMMA 2.4. If $A$ is $S P D$, then $B A$ is A-self-adjoint if and only if $B$ is selfadjoint.

Proof. Note that $(A B A u, v)=(B A u, A v)=\left(A u, B^{T} A v\right)$. The lemma follows since $B A=B^{T} A$ if and only if $B=B^{T}$.

LEMMA 2.5. If $A$ is $S P D$, then $E$ is $A$-self-adjoint if and only if $B$ is self-adjoint.

Proof. Note that: $(A E u, v)=(A u, v)-(A B A u, v)=(A u, v)-\left(A u,(B A)^{*} v\right)=$ $\left(A u,\left(I-(B A)^{*}\right) v\right)$. Therefore, $E^{*}=E$ if and only if $B A=(B A)^{*}$. By Lemma 2.4, this holds if and only if $B$ is self-adjoint.

LEMma 2.6. If $A$ and $B$ are $S P D$, then $B A$ is $A-S P D$.

Proof. By Lemma 2.4, $B A$ is $A$-self-adjoint. Also, $(A B A u, u)=(B A u, A u)=$ $\left(B^{1 / 2} A u, B^{1 / 2} A u\right)>0 \forall u \neq 0$. Hence, $B A$ is $A$-positive, and the result follows.

LEMma 2.7. If $A$ is SPD and $B$ is self-adjoint, then $\|E\|_{A}=\rho(E)$.

Proof. By Lemma 2.5, $E$ is $A$-self-adjoint. By Lemma 2.1, the result follows.

LEMMA 2.8. If $E^{*}$ is the A-adjoint of $E$, then $\|E\|_{A}^{2}=\left\|E E^{*}\right\|_{A}$. 
Proof. The proof follows that of a familiar result for the Euclidean 2-norm [12].

LEMMA 2.9. If $A$ and $B$ are $S P D$, and $E$ is A-nonnegative, then $\|E\|_{A}<1$.

Proof. By Lemma 2.5, $E$ is $A$-self-adjoint. $A s E$ is $A$-nonnegative, it holds that $(E u, u)_{A} \geq 0$, or $(B A u, u)_{A} \leq(u, u)_{A}$. By Lemma 2.6, $B A$ is $A$-SPD, and we have that $0<(B A u, u)_{A} \leq(u, u)_{A} \forall u \neq 0$, which, by (3), implies that $0<\lambda_{i} \leq$ $1 \forall \lambda_{i} \in \sigma(B A)$. Thus, $\rho(E)=1-\min _{i} \lambda_{i}<1$. Finally, by Lemma 2.7 , we have $\|E\|_{A}=\rho(E)$.

We will also have use for the following two simple lemmas.

LEMMA 2.10. If $A$ is $S P D$ and $B$ is self-adjoint, and $E$ is such that

$$
-C_{1}(u, u)_{A} \leq(E u, u)_{A} \leq C_{2}(u, u)_{A} \quad \forall u \in \mathcal{H},
$$

for $C_{1} \geq 0$ and $C_{2} \geq 0$, then $\rho(E)=\|E\|_{A} \leq \max \left\{C_{1}, C_{2}\right\}$.

Proof. By Lemma 2.5, $E$ is $A$-self-adjoint, and by $(3), \lambda_{\min }(E)$ and $\lambda_{\max }(E)$ are bounded by $-C_{1}$ and $C_{2}$, respectively. The result then follows by Lemma 2.7 .

LEMMA 2.11. If $A$ and $B$ are $S P D$, then Lemma 2.10 holds for some $C_{2}<1$.

Proof. By Lemma 2.6, $B A$ is $A$-SPD, which implies that the eigenvalues of $B A$ are real and positive. Hence, we must have that $\lambda_{i}(E)=1-\lambda_{i}(B A)<1 \forall i$. Since $C_{2}$ in Lemma 2.10 bounds the largest positive eigenvalue of $E$, we have that $C_{2}<1$.

2.3. Krylov acceleration of SPD linear methods. The conjugate gradient method was developed by Hestenes and Stiefel [13] as a method for solving linear systems $A u=f$ with SPD operators $A$. In order to improve convergence, it is common to precondition the linear system by an SPD preconditioning operator $B \approx A^{-1}$, in which case the generalized or preconditioned conjugate gradient method results [8]. Our goal in this section is to briefly review some relationships between the contraction number of a basic linear preconditioner and that of the resulting preconditioned conjugate gradient algorithm.

We start with the well-known conjugate gradient contraction bound [12]

$$
\left\|e^{i+1}\right\|_{A} \leq 2\left(1-\frac{2}{1+\sqrt{\kappa_{A}(B A)}}\right)^{i+1}\left\|e^{0}\right\|_{A}=2 \delta_{\mathrm{cg}}^{i+1}\left\|e^{0}\right\|_{A} .
$$

The ratio of extreme eigenvalues of $B A$ appearing in the derivation of the bound gives rise to the generalized condition number $\kappa_{A}(B A)$ appearing above. This ratio is often mistakenly called the (spectral) condition number $\kappa(B A)$; in fact, since $B A$ is not self-adjoint, this ratio is not in general equal to the usual condition number (this point is discussed in great detail in [1]). However, the ratio does yield a condition number in the $A$-norm. The following lemma is a special case of Corollary 4.2 in [1].

LEMMA 2.12. If $A$ and $B$ are $S P D$, then

$$
\kappa_{A}(B A)=\|B A\|_{A}\left\|(B A)^{-1}\right\|_{A}=\frac{\lambda_{\max }(B A)}{\lambda_{\min }(B A)} .
$$

Remark 2.1. Often a linear method requires a parameter $\alpha$ in order to be convergent, leading to an error propagator of the form $E=I-\alpha B A$. Equation (7) shows that the $A$-condition number does not depend on the particular choice of $\alpha$. Hence, one can use the conjugate gradient method as an accelerator for the method without a parameter, avoiding the possibly costly estimation of a good $\alpha$. 
The following result gives a bound on the condition number of the operator $B A$ in terms of the extreme eigenvalues of the error propagator $E=I-B A$; such bounds are often used in the analysis of linear preconditioners (cf. Proposition 5.1 in [26]). We give a short proof of this result for completeness.

LEMma 2.13. If $A$ and $B$ are $S P D$, and $E$ is such that

$$
-C_{1}(u, u)_{A} \leq(E u, u)_{A} \leq C_{2}(u, u)_{A} \quad \forall u \in \mathcal{H},
$$

for $C_{1} \geq 0$ and $C_{2} \geq 0$, then the above must hold with $C_{2}<1$, and it follows that

$$
\kappa_{A}(B A) \leq \frac{1+C_{1}}{1-C_{2}}
$$

Proof. First, since $A$ and $B$ are SPD, by Lemma 2.11 we have that $C_{2}<1$. Since $(E u, u)_{A}=(u, u)_{A}-(B A u, u)_{A}$, it is clear that

$$
\left(1-C_{2}\right)(u, u)_{A} \leq(B A u, u)_{A} \leq\left(1+C_{1}\right)(u, u)_{A} \quad \forall u \in \mathcal{H} .
$$

By Lemma 2.6, $B A$ is $A$-SPD. Its eigenvalues are real and positive and lie in the interval defined by the Rayleigh quotients generated by the $A$-inner-product. Hence, that interval is given by $\left[\left(1-C_{2}\right),\left(1+C_{1}\right)\right]$, and by Lemma 2.12 the result follows.

Remark 2.2. Even if a linear method is not convergent, it may still be a good preconditioner. If it is the case that $C_{2} \ll 1$, and if $C_{1}>1$ does not become too large, then $\kappa_{A}(B A)$ will be small and the conjugate gradient method will converge rapidly, even though the linear method diverges.

If only a bound on the norm of the error propagator $E=I-B A$ is available, then the following result can be used to bound the condition number of $B A$. This result is used, for example, in [27].

COROLlary 2.14. If $A$ and $B$ are $S P D$, and $\|I-B A\|_{A} \leq \delta<1$, then

$$
\kappa_{A}(B A) \leq \frac{1+\delta}{1-\delta}
$$

Proof. This follows immediately from Lemma 2.13 with $\delta=\max \left\{C_{1}, C_{2}\right\}$.

The next result connects the contraction number of the preconditioner to the contraction number of the preconditioned conjugate gradient method. It shows that the conjugate gradient method always accelerates a linear method (if the conditions of the lemma hold).

LEMmA 2.15. If $A$ and $B$ are $S P D$, and $\|I-B A\|_{A} \leq \delta<1$, then $\delta_{\mathrm{cg}}<\delta$.

Proof. An abbreviated proof appears in [27]; a more detailed proof appears in [14].

2.4. Krylov acceleration of nonsymmetric linear methods. The convergence theory of the conjugate gradient iteration requires that the preconditioned operator $B A$ be $A$-self-adjoint (see [2] for more general conditions), which from Lemma 2.4 requires that $B$ be self-adjoint. If a Schwarz method is employed which produces a nonsymmetric operator $B$, then although $A$ is $\mathrm{SPD}$, the theory of the previous section does not apply, and a nonsymmetric solver such as conjugate gradients on the normal equations [2], GMRES [21], CGS [23], or Bi-CGstab [25] must be used for the now non- $A$-SPD preconditioned system $B A u=B f$.

The conjugate gradient method for SPD problems has several nice properties (good convergence rate, efficient three-term recursion, and minimization of the $A$ norm of the error at each step), some of which must be given up in order to generalize 
the method to nonsymmetric problems. For example, while GMRES attempts to maintain a minimization property and a good convergence rate, the three-term recursion must be sacrificed. Conjugate gradients on the normal equations maintains a minimization property as well as the efficient three-term recursion but sacrifices convergence speed (the effective condition number is the square of the original system). Methods such as CGS and Bi-CGstab sacrifice the minimization property but maintain good convergence speed and the efficient three-term recursion. For these reasons, methods such as CGS and Bi-CGstab have become the methods of choice in many applications that give rise to nonsymmetric problems. Bi-CGstab has been shown to be more attractive than CGS in many situations due to the more regular convergence behavior [25]. In addition, Bi-CGstab does not require the application of the adjoint of the preconditioning operator, which can be difficult to implement in the case of some Schwarz methods.

In section 6, we shall use the preconditioned Bi-CGstab algorithm to accelerate nonsymmetric Schwarz methods. In a sequence of numerical experiments, we shall compare the effectiveness of this approach with unaccelerated symmetric and nonsymmetric Schwarz methods and with symmetric Schwarz methods accelerated with conjugate gradients.

3. Multiplicative Schwarz methods. We develop a preconditioning theory of product algorithms which establishes sufficient conditions for producing SPD preconditioners. This theory is used to establish sufficient SPD conditions for multiplicative DD and MG methods.

3.1. A product operator. Consider a product operator of the form

$$
E=I-B A=\left(I-\bar{B}_{1} A\right)\left(I-B_{0} A\right)\left(I-B_{1} A\right),
$$

where $\bar{B}_{1}, B_{0}$, and $B_{1}$ are linear operators on $\mathcal{H}$, and where $A$ is, as before, an SPD operator on $\mathcal{H}$. We are interested in conditions for $\bar{B}_{1}, B_{0}$, and $B_{1}$, which guarantee that the implicitly defined operator $B$ is self-adjoint and positive definite and, hence, can be accelerated by using the conjugate gradient method.

LEMMA 3.1. Sufficient conditions for symmetry and positivity of operator B, implicitly defined by (10), are as follows:

1. $\bar{B}_{1}=B_{1}^{T}$;

2. $B_{0}=B_{0}^{T}$;

3. $\left\|I-B_{1} A\right\|_{A}<1$;

4. $B_{0}$ nonnegative on $\mathcal{H}$.

Proof. By Lemma 2.5, in order to prove symmetry of $B$, it is sufficient to prove that $E$ is $A$-self-adjoint. By using (1), we get

$$
\begin{aligned}
E^{*} & =A^{-1} E^{T} A \\
& =A^{-1}\left(I-A B_{1}^{T}\right)\left(I-A B_{0}^{T}\right)\left(I-A \bar{B}_{1}^{T}\right) A \\
& =\left(I-B_{1}^{T} A\right)\left(I-B_{0}^{T} A\right)\left(I-\bar{B}_{1}^{T} A\right) \\
& =\left(I-\bar{B}_{1} A\right)\left(I-B_{0} A\right)\left(I-B_{1} A\right)=E,
\end{aligned}
$$

which follows from conditions 1 and 2 .

Next, we prove that $(B u, u)>0 \forall u \in \mathcal{H}, u \neq 0$. Since $A$ is nonsingular, this is equivalent to proving that $(B A u, A u)>0$. Using condition 1 , we have that

$$
\begin{aligned}
(B A u, A u) & =((I-E) u, A u) \\
& =(u, A u)-\left(\left(I-B_{1}^{T} A\right)\left(I-B_{0} A\right)\left(I-B_{1} A\right) u, A u\right)
\end{aligned}
$$




$$
\begin{aligned}
& =(u, A u)-\left(\left(I-B_{0} A\right)\left(I-B_{1} A\right) u, A\left(I-B_{1} A\right) u\right) \\
& =(u, A u)-\left(\left(I-B_{1} A\right) u, A\left(I-B_{1} A\right) u\right)+\left(B_{0} w, w\right),
\end{aligned}
$$

where $w=A\left(I-B_{1} A\right) u$. By condition 4 , we have that $\left(B_{0} w, w\right) \geq 0$. Condition 3 implies that $\left(\left(I-B_{1} A\right) u, A\left(I-B_{1} A\right) u\right)<(u, A u)$ for $u \neq 0$. Thus, the first two terms in the sum above are together positive, while the third one is nonnegative, so that $B$ is positive.

Corollary 3.2. If $B_{1}=B_{1}^{T}$, then condition 3 in Lemma 3.1 is equivalent to $\rho\left(I-B_{1} A\right)<1$.

Proof. This follows directly from Lemma 2.1 and Lemma 2.5.

3.2. Multiplicative DD. Given the finite-dimensional Hilbert space $\mathcal{H}$, consider $J$ spaces $\mathcal{H}_{k}, \quad k=1, \ldots, J$, together with linear operators $I_{k} \in \mathbf{L}\left(\mathcal{H}_{k}, \mathcal{H}\right)$, $\operatorname{null}\left(I_{k}\right)=\{0\}$, such that $I_{k} \mathcal{H}_{k} \subseteq \mathcal{H}=\sum_{k=1}^{J} I_{k} \mathcal{H}_{k}$. We also assume the existence of another space $\mathcal{H}_{0}$, an associated operator $I_{0}$ such that $I_{0} \mathcal{H}_{0} \subseteq \mathcal{H}$, and some linear operators $I^{k} \in \mathbf{L}\left(\mathcal{H}, \mathcal{H}_{k}\right), k=0, \ldots, J$. For notational convenience, we shall denote the inner-products on $\mathcal{H}_{k}$ by $(\cdot, \cdot)$ (without explicit reference to the particular space). Note that the inner-products on different spaces need not be related.

In a DD context, the spaces $\mathcal{H}_{k}, k=1, \ldots, J$, are typically associated with local subdomains of the original domain on which the partial differential equation is defined. The space $\mathcal{H}_{0}$ is then a space associated with some global coarse mesh. The operators $I_{k}, k=1, \ldots, J$, are usually inclusion operators, while $I_{0}$ is an interpolation or prolongation operator (as in a two-level MG method). The operators $I^{k}, k=$ $1, \ldots, J$, are usually orthogonal projection operators, while $I^{0}$ is a restriction operator (again, as in a two-level MG method).

The error propagator of a multiplicative DD method on the space $\mathcal{H}$ employing the subspaces $I_{k} \mathcal{H}_{k}$ has the general form [9]

$$
E=I-B A=\left(I-I_{J} \bar{R}_{J} I^{J} A\right) \cdots\left(I-I_{0} R_{0} I^{0} A\right) \cdots\left(I-I_{J} R_{J} I^{J} A\right),
$$

where $\bar{R}_{k}$ and $R_{k}, k=1, \ldots, J$, are linear operators on $\mathcal{H}_{k}$, and $R_{0}$ is a linear operator on $\mathcal{H}_{0}$. Usually the operators $\bar{R}_{k}$ and $R_{k}$ are constructed so that $\bar{R}_{k} \approx A_{k}^{-1}$ and $R_{k} \approx A_{k}^{-1}$, where $A_{k}$ is the operator defining the subdomain problem in $\mathcal{H}_{k}$. Similarly, $R_{0}$ is constructed so that $R_{0} \approx A_{0}^{-1}$. Actually, quite often $R_{0}$ is a "direct solve," i.e., $R_{0}=A_{0}^{-1}$. The subdomain problem operator $A_{k}$ is related to the restriction of $A$ to $\mathcal{H}_{k}$. We say that $A_{k}$ satisfies the Galerkin conditions or, in a finite element setting, that it is variationally defined when

$$
A_{k}=I^{k} A I_{k}, \quad I^{k}=I_{k}^{T} .
$$

Recall that the superscript " $T$ " is to be interpreted as the adjoint in the sense of (2), i.e., with respect to the inner-products in $\mathcal{H}$ and $\mathcal{H}_{k}$.

In the case of finite element, finite volume, or finite difference discretization of an elliptic problem, conditions (12) can be shown to hold naturally for both the matrices and the abstract weak form operators for all subdomains $k=1, \ldots, J$. For the coarse space $\mathcal{H}_{0}$, often (12) must be imposed algebraically.

Propagator (11) can be thought of as the product operator (10), by choosing

$$
I-\bar{B}_{1} A=\prod_{k=J}^{1}\left(I-I_{k} \bar{R}_{k} I^{k} A\right), \quad B_{0}=I_{0} R_{0} I^{0}, \quad I-B_{1} A=\prod_{k=1}^{J}\left(I-I_{k} R_{k} I^{k} A\right),
$$


where $\bar{B}_{1}$ and $B_{1}$ are known only implicitly. (Note that we take the convention that the first term in the product appears on the left.) This identification allows for the use of Lemma 3.1 to establish sufficient conditions on the subdomain operators $\bar{R}_{k}$, $R_{k}$, and $R_{0}$ to guarantee that multiplicative DD yields an SPD operator $B$.

THEOREM 3.3. Sufficient conditions for symmetry and positivity of the multiplicative $D D$ operator $B$, implicitly defined by (11), are as follows:

1. $I^{k}=c_{k} I_{k}^{T}, \quad c_{k}>0, \quad k=0, \ldots, J$;

2. $\bar{R}_{k}=R_{k}^{T}, \quad k=1, \ldots, J$;

3. $R_{0}=R_{0}^{T}$;

4. $\left\|\prod_{k=1}^{J}\left(I-I_{k} R_{k} I^{k} A\right)\right\|_{A}<1$;

5. $R_{0}$ nonnegative on $\mathcal{H}_{0}$.

Proof. We show that the sufficient conditions of Lemma 3.1 are satisfied. First, we prove that $\bar{B}_{1}=B_{1}^{T}$, which, by Lemma 2.5 , is equivalent to proving that $\left(I-B_{1} A\right)^{*}=$ $\left(I-\bar{B}_{1} A\right)$. By using (1), we have

$$
\left(\prod_{k=1}^{J}\left(I-I_{k} R_{k} I^{k} A\right)\right)^{*}=A^{-1}\left(\prod_{k=1}^{J}\left(I-I_{k} R_{k} I^{k} A\right)\right)^{T} A=\prod_{k=J}^{1}\left(I-\left(I^{k}\right)^{T} R_{k}^{T}\left(I_{k}\right)^{T} A\right),
$$

which equals $\left(I-\bar{B}_{1} A\right)$ under conditions 1 and 2 of the theorem. The symmetry of $B_{0}$ follows immediately from conditions 1 and 3 ; indeed,

$$
B_{0}^{T}=\left(I_{0} R_{0} I^{0}\right)^{T}=\left(I^{0}\right)^{T} R_{0}^{T}\left(I_{0}\right)^{T}=\left(c_{0} I_{0}\right) R_{0}\left(c_{0}^{-1} I^{0}\right)=I_{0} R_{0} I^{0}=B_{0} .
$$

By condition 4 of the theorem, condition 3 of Lemma 3.1 holds trivially. The theorem follows by realizing that condition 4 of Lemma 3.1 is also satisfied, since

$$
\left(B_{0} u, u\right)=\left(I_{0} R_{0} I^{0} u, u\right)=\left(R_{0} I^{0} u, I_{0}^{T} u\right)=c_{0}^{-1}\left(R_{0} I^{0} u, I^{0} u\right) \geq 0 \quad \forall u \in \mathcal{H} .
$$

Remark 3.3. Note that one sweep through the subdomains, followed by a coarse problem solve, followed by another sweep through the subdomains in reversed order, gives rise to an error propagator of the form (11). Also, note that no conditions are imposed on the nature of the operators $A_{k}$ associated with each subdomain. In particular, the theorem does not require that the variational conditions are satisfied. While it is natural for condition (12) to hold between the fine space and the spaces associated with each subdomain, these conditions are often difficult to enforce for the coarse problem. Violation of variational conditions can occur, for example, when complex coefficient discontinuities do not lie along element boundaries on the coarse mesh (we present numerical results for such a problem in section 6). The theorem also does not require that the overall multiplicative DD method be convergent.

Remark 3.4. The results of the theorem apply for abstract operators on general finite-dimensional Hilbert spaces with arbitrary inner-products. They hold in particular for matrix operators on $\mathbb{R}^{n}$, equipped with the Euclidean inner-product, or the discrete $L^{2}$ inner-product. In the former case, the superscript " $T$ " corresponds to the standard matrix transpose. In the latter case, the matrix representation of the adjoint is a scalar multiple of the matrix transpose; the scalar may be different from unity when the adjoint involves two different spaces, as in the case of prolongation and restriction. This possible constant in the case of the discrete $L^{2}$ inner-product is absorbed in the factor $c_{k}$ in condition 1. This allows for an easy verification of the conditions of the theorem in an actual implementation, where the operators are 
represented as matrices and where the inner-products do not explicitly appear in the algorithm.

Remark 3.5. Condition 1 of the theorem (with $c_{k}=1$ ) for $k=1, \ldots, J$ is usually satisfied trivially for DD methods. For $k=0$, it may have to be imposed explicitly. Condition 2 of the theorem allows for several alternatives which give rise to an SPD preconditioner, namely, (1) use of exact subdomain solvers (if $A_{k}$ is a symmetric operator); (2) use of identical symmetric subdomain solvers in the forward and backward sweeps; and (3) use of the adjoint of the subdomain solver on the second sweep. Condition 3 is satisfied when the coarse problem is symmetric and the solve is an exact one, which is usually the case. If not, the coarse problem solve has to be symmetric. Condition 4 in Theorem 3.3 is clearly a nontrivial one; it is essentially the assumption that the multiplicative DD method without a coarse space is convergent. Convergence theories for DD methods can be quite technical and depend on such things as the discretization, the subdomain number, shape, and size, and the regularity of the solution $[5,9,27]$. However, since variational conditions hold naturally between the fine space and each subdomain space for nearly any formulation of a DD method, very general convergence theorems can be derived, if one is not concerned about the actual rate of convergence. Using the Schwarz theory framework in any of [5, 9, 27], it can be shown that condition 4 in Theorem 3.3 (convergence of multiplicative DD without a coarse space) holds if the variational conditions (12) hold, and if the subdomain solvers $R_{k}$ are SPD. A proof of this result may be found, for example, in [14]. Condition 5 is satisfied, for example, when the coarse problem is SPD and the solve is exact.

Consider now the case when the subspaces together do not span the entire space, except when the coarse space is included. The above theorem can be applied with $R_{0}=0$, and by viewing the coarse space as simply one of the spaces $\mathcal{H}_{k}, k \neq 0$. In this case, the error propagation operator $E$ takes the form

$$
I-B A=\left(I-I_{J} \bar{R}_{J} I^{J} A\right) \cdots\left(I-I_{1} \bar{R}_{1} I^{1} A\right)\left(I-I_{1} R_{1} I^{1} A\right) \cdots\left(I-I_{J} R_{J} I^{J} A\right) .
$$

This leads to the following corollary.

COROLLARY 3.4. Sufficient conditions for symmetry and positivity of the multiplicative DD operator $B$, implicitly defined by (13), are as follows:

1. $I^{k}=c_{k} I_{k}^{T}, \quad c_{k}>0, \quad k=1, \ldots, J$;

2. $\bar{R}_{k}=R_{k}^{T}, \quad k=1, \ldots, J$

3. $\left\|\prod_{k=1}^{J}\left(I-I_{k} R_{k} I^{k} A\right)\right\|_{A}<1$.

Remark 3.6. Condition 3 is equivalent to requiring convergence of the overall multiplicative Schwarz method. This follows from the relationship

$$
\|E\|_{A}=\left\|\bar{E}^{*} \bar{E}\right\|_{A}=\|\bar{E}\|_{A}^{2}<1,
$$

where $\bar{E}=\prod_{k=1}^{J}\left(I-I_{k} R_{k} I^{k} A\right)$.

Remark 3.7. If, in addition to the conditions of the corollary, it holds that $R_{1}=$ $\left(I^{1} A I_{1}\right)^{-1}$, i.e., it corresponds to an exact solve with a variationally defined subspace problem operator in the sense of (12), then

$$
\left(I-I_{1} \bar{R}_{1} I^{1} A\right)\left(I-I_{1} R_{1} I^{1} A\right)=I-I_{1} R_{1} I^{1} A,
$$

since $I-I_{1}\left(I^{1} A I_{1}\right)^{-1} I_{1} A$ is a projector. Therefore, space $\mathcal{H}_{1}$ (for example, the coarse space) needs to be visited only once in the application of (13). 
3.3. Multiplicative MG. Consider the Hilbert space $\mathcal{H}, J$ spaces $\mathcal{H}_{k}$ together with linear operators $I_{k} \in \mathbf{L}\left(\mathcal{H}_{k}, \mathcal{H}\right)$, $\operatorname{null}\left(I_{k}\right)=0$, such that the spaces $I_{k} \mathcal{H}_{k}$ are nested and satisfy $I_{1} \mathcal{H}_{1} \subseteq I_{2} \mathcal{H}_{2} \subseteq \cdots \subseteq I_{J-1} \mathcal{H}_{J-1} \subseteq \mathcal{H}_{J} \equiv \mathcal{H}$. As before we denote the $\mathcal{H}_{k}$-inner-products by $(\cdot, \cdot)$, since it will be clear from the arguments which innerproduct is intended. Again, the inner-products are not necessarily related in any way. We assume also the existence of operators $I^{k} \in \mathbf{L}\left(\mathcal{H}, \mathcal{H}_{k}\right)$.

In a MG context, the spaces $\mathcal{H}_{k}$ are typically associated with a nested hierarchy of successively refined meshes, with $\mathcal{H}_{1}$ being the coarsest mesh and $\mathcal{H}_{J}$ being the fine mesh on which the partial differential equation solution is desired. The linear operators $I_{k}$ are prolongation operators, constructed from given interpolation or prolongation operators that operate between subspaces, i.e., $I_{k-1}^{k} \in \mathbf{L}\left(\mathcal{H}_{k-1}, \mathcal{H}_{k}\right)$. The operator $I_{k}$ is then constructed (only as a theoretical tool) as a composite operator

$$
I_{k}=I_{J-1}^{J} I_{J-2}^{J-1} \cdots I_{k+1}^{k+2} I_{k}^{k+1}, \quad k=1, \ldots, J-1 .
$$

The composite restriction operators $I^{k}, k=1, \ldots, J-1$, are constructed similarly from some given restriction operators $I_{k}^{k-1} \in \mathbf{L}\left(\mathcal{H}_{k}, \mathcal{H}_{k-1}\right)$.

The coarse problem operators $A_{k}$ are related to the restriction of $A$ to $\mathcal{H}_{k}$. As in the case of DD methods, we say that $A_{k}$ is variationally defined or satisfies the Galerkin conditions when conditions (12) hold. It is not difficult to see that conditions (12) are equivalent to the recursively defined variational conditions

$$
A_{k}=I_{k+1}^{k} A_{k+1} I_{k}^{k+1}, \quad I_{k+1}^{k}=\left(I_{k}^{k+1}\right)^{T}
$$

when the composite operators $I_{k}$ appearing in (12) are defined as in (14).

In a finite element setting, conditions (15) can be shown to hold in ideal situations, for both the stiffness matrices and the abstract weak form operators, for a nested sequence of successively refined finite element meshes. In the finite difference or finite volume method setting, conditions (15) must often be imposed algebraically, in a recursive fashion.

The error propagator of a multiplicative V-cycle MG method is defined implicitly:

$$
E=I-B A=I-D_{J} A_{J},
$$

where $A_{J}=A$ and where operators $D_{k}, k=2, \ldots, J$ are defined recursively,

$$
\begin{aligned}
I-D_{k} A_{k} & =\left(I-\bar{R}_{k} A_{k}\right)\left(I-I_{k-1}^{k} D_{k-1} I_{k}^{k-1} A_{k}\right)\left(I-R_{k} A_{k}\right), k=2, \ldots, J, \\
D_{1} & =R_{1} .
\end{aligned}
$$

Operators $\bar{R}_{k}$ and $R_{k}$ are linear operators on $\mathcal{H}_{k}$, usually called smoothers. The linear operators $A_{k} \in L\left(\mathcal{H}_{k}, \mathcal{H}_{k}\right)$ define the coarse problems. They often satisfy the variational condition (15).

The error propagator (16) can be thought of as an operator of the form (10) with

$$
\bar{B}_{1}=\bar{R}_{J}, \quad B_{0}=I_{J-1}^{J} D_{J-1} I_{J}^{J-1}, B_{1}=R_{J} .
$$

Such an identification with the product method allows for the use of the result in Lemma 3.1. The following theorem establishes sufficient conditions for the subspace operators $R_{k}, \bar{R}_{k}$, and $A_{k}$ in order to generate an (implicitly defined) SPD operator $B$ that can be accelerated with conjugate gradients.

THEOREM 3.5. Sufficient conditions for symmetry and positivity of the multiplicative $M G$ operator B, implicitly defined by (16), (17), and (18), are as follows: 
1. $A_{k}$ is $S P D$ on $\mathcal{H}_{k}, \quad k=2, \ldots, J$;

2. $I_{k}^{k-1}=c_{k}\left(I_{k-1}^{k}\right)^{T}, \quad c_{k}>0, \quad k=2, \ldots, J$;

3. $\bar{R}_{k}=R_{k}^{T}, \quad k=2, \ldots, J$;

4. $R_{1}=R_{1}^{T}$

5. $\left\|I-R_{J} A\right\|_{A}<1$

6. $\left\|I-R_{k} A_{k}\right\|_{A_{k}} \leq 1, \quad k=2, \ldots, J-1$;

7. $R_{1}$ nonnegative on $\mathcal{H}_{1}$.

Proof. Since $\bar{R}_{J}=R_{J}^{T}$, we have that $\bar{B}_{1}=B_{1}^{T}$, which gives condition 1 of Lemma 3.1. Now, $B_{0}$ is symmetric if and only if

$$
B_{0}=I_{J-1}^{J} D_{J-1} I_{J}^{J-1}=\left(c_{J}^{-1} I_{J}^{J-1}\right)^{T} D_{J-1}^{T}\left(c_{J} I_{J-1}^{J}\right)^{T}=B_{0}^{T},
$$

which holds under condition 2 and a symmetry requirement for $D_{J-1}$. We will prove that $D_{J-1}=D_{J-1}^{T}$ by induction. First, $D_{1}=D_{1}^{T}$ since $R_{1}=R_{1}^{T}$. By Lemma 2.5 and condition $1, D_{k}$ is symmetric if and only if $E_{k}=I-D_{k} A_{k}$ is $A_{k}$-self-adjoint. By using (1), we have that

$$
\begin{aligned}
E_{k}^{*} & =A_{k}^{-1}\left(\left(I-\bar{R}_{k} A_{k}\right)\left(I-I_{k-1}^{k} D_{k-1} I_{k}^{k-1} A_{k}\right)\left(I-R_{k} A_{k}\right)\right)^{T} A_{k} \\
& =A_{k}^{-1}\left(I-A_{k}^{T} R_{k}^{T}\right)\left(I-A_{k}^{T}\left(I_{k}^{k-1}\right)^{T} D_{k-1}^{T}\left(I_{k-1}^{k}\right)^{T}\right)\left(I-A_{k}^{T} \bar{R}_{k}^{T}\right) A_{k} \\
& =\left(I-R_{k}^{T} A_{k}\right) A_{k}^{-1}\left(I-A_{k}^{T}\left(I_{k}^{k-1}\right)^{T} D_{k-1}^{T}\left(I_{k-1}^{k}\right)^{T}\right) A_{k}\left(I-\bar{R}_{k}^{T} A_{k}\right) \\
& =\left(I-\bar{R}_{k} A_{k}\right)\left(I-\left(c_{k} I_{k-1}^{k}\right) D_{k-1}^{T}\left(c_{k}^{-1} I_{k}^{k-1}\right) A_{k}\right)\left(I-R_{k} A_{k}\right),
\end{aligned}
$$

where we have used conditions 1,2 , and 3 . Therefore, $E_{k}^{*}=E_{k}$, if $D_{k-1}=D_{k-1}^{T}$. Hence, the result follows by induction on $k$.

Condition 3 of Lemma 3.1 follows trivially by condition 5 of the theorem.

It remains to verify condition 4 of Lemma 3.1 , namely, that $B_{0}$ is nonnegative. This is equivalent to showing that $D_{J-1}$ is nonnegative on $\mathcal{H}_{J-1}$. This will follow again from an induction argument. First, note that $D_{1}=R_{1}$ is nonnegative on $\mathcal{H}_{1}$. Next, we prove that $\left(D_{k} v_{k}, v_{k}\right) \geq 0 \forall v_{k} \in \mathcal{H}_{k}$, or, equivalently, since $A_{k}$ is nonsingular, that $\left(D_{k} A_{k} v_{k}, A_{k} v_{k}\right) \geq 0$. So, $\forall v_{k} \in \mathcal{H}_{k}$,

$$
\begin{aligned}
\left(D_{k} A_{k} v_{k}, A_{k} v_{k}\right)= & \left(A_{k} v_{k}, v_{k}\right)-\left(A_{k} E_{k} v_{k}, v_{k}\right) \\
= & \left(A_{k} v_{k}, v_{k}\right)-\left(A_{k}\left(I-\bar{R}_{k} A_{k}\right)\left(I-I_{k-1}^{k} D_{k-1} I_{k}^{k-1} A_{k}\right)\left(I-R_{k} A_{k}\right) v_{k}, v_{k}\right) \\
= & \left(A_{k} v_{k}, v_{k}\right)-\left(A_{k}\left(I-I_{k-1}^{k} D_{k-1} I_{k}^{k-1} A_{k}\right)\left(I-R_{k} A_{k}\right) v_{k},\left(I-R_{k} A_{k}\right) v_{k}\right) \\
= & \left(A_{k} v_{k}, v_{k}\right)-\left(A_{k}\left(I-R_{k} A_{k}\right) v_{k},\left(I-R_{k} A_{k}\right) v_{k}\right) \\
& +\left(A_{k} I_{k-1}^{k} D_{k-1} I_{k}^{k-1} A_{k}\left(I-R_{k} A_{k}\right) v_{k},\left(I-R_{k} A_{k}\right) v_{k}\right) \\
= & \left(v_{k}, v_{k}\right)_{A_{k}}-\left(S_{k} v_{k}, S_{k} v_{k}\right)_{A_{k}}+c_{k}^{-1}\left(D_{k-1} v_{k-1}, v_{k-1}\right),
\end{aligned}
$$

where $S_{k}=I-R_{k} A_{k}$ and $v_{k-1}=I_{k}^{k-1} A_{k}\left(I-R_{k} A_{k}\right) v_{k} \in \mathcal{H}_{k-1}$. By condition 6 , the first two terms in the above sum add up to a nonnegative value. Hence, $D_{k}$ is nonnegative if $D_{k-1}$ is nonnegative. Condition 4 of Lemma 3.1 follows.

COROLLARY 3.6. If the fine grid smoother is symmetric, i.e., $R_{J}=\bar{R}_{J}^{T}$, then condition 5 in Theorem 3.5 is equivalent to $\rho\left(I-R_{J} A\right)<1$.

Proof. This follows directly from Corollary 3.2.

Remark 3.8. The coarse grid operators $A_{k}, k=2, \ldots, J-1$, need only be SPD. They need not satisfy the Galerkin conditions (15).

Remark 3.9. As noted earlier in Remark 3.4, the conditions and conclusions of the theorem can be interpreted completely in terms of the usual matrix representations of the MG operators. 
Remark 3.10. Condition 1 of the theorem requires that the coarse grid operators (except for the coarsest one) be SPD. This is easily satisfied when they are constructed either by discretization or by explicitly using the Galerkin or variational condition. Condition 2 requires restriction and prolongation to be adjoints, possibly multiplied by an arbitrary constant. Condition 3 of the theorem is satisfied when the number of presmoothing steps equals the number of postsmoothing steps, and, in addition, one of the following is imposed: (1) use of the same symmetric smoother for both presmoothing and postsmoothing; (2) use of the adjoint of the presmoothing operator as the postsmoother. Condition 4 requires a symmetric coarsest mesh solver. When the coarsest mesh problem is $\mathrm{SPD}$, the symmetry of $R_{1}$ is satisfied when it corresponds to an exact solve (as is typical for MG methods). Condition 5 is a convergence requirement on the fine space smoother. Condition 6 requires the coarse grid smoothers to be nondivergent. The nonnegativity requirement for $R_{1}$ is a nontrivial one; however, if $A_{1}$ is SPD, it is immediately satisfied when the operator corresponds to an exact solve.

Theorem 3.5 applies to standard MG methods only. The conditions of the theorem, and condition 5 in particular, cannot be satisfied in the cases of hierarchical basis MG methods [3] and MG methods with local smoothing on locally refined regions. The latter methods are covered in the following theorem, where the conditions that guarantee positivity of the preconditioner (conditions 5, 6, and 7 in Theorem 3.5) are replaced by a convergence condition on the underlying iterative method.

THEOREM 3.7. Sufficient conditions for symmetry and positivity of the multiplicative $M G$ operator $B$, implicitly defined by (16), (17), and (18), are as follows:

1. $A_{k}$ is $S P D$ on $\mathcal{H}_{k}, \quad k=2, \ldots, J$;

2. $I_{k}^{k-1}=c_{k}\left(I_{k-1}^{k}\right)^{T}, \quad c_{k}>0, \quad k=2, \ldots, J$;

3. $\bar{R}_{k}=R_{k}^{T}, \quad k=2, \ldots, J$;

4. $R_{1}=R_{1}^{T}$

5. $\|I-B A\|_{A}<1$.

Proof. Positivity of $B$ is proven easily by a contradiction argument. Symmetry follows from the proof of Theorem 3.5.

Requiring convergence of the underlying MG method is very restrictive; it is not a necessary condition. Positivity of $B$ is satisfied if $\lambda_{i}(I-B A)<1$; no limit needs to be set on the magnitude of the negative eigenvalues. The above eigenvalue condition, however, does not seem to lead to conditions that are easily checked in practice.

Remark 3.11. If variational conditions are satisfied on all levels, then there is a simple proof which shows that in addition to defining an SPD operator $B$, the conditions of Theorem 3.5 are sufficient to prove the convergence of the MG method itself. The result is as follows.

THEOREM 3.8. If in addition to the conditions for Theorem 3.5 it holds that $A_{k}=I^{k} A I_{k}, I^{k}=I_{k}^{T}$, and $R_{1}=A_{1}^{-1}$, then the $M G$ error propagator satisfies

$$
\rho(E)=\|E\|_{A}<1 .
$$

Proof. Under the conditions of the theorem, the MG error propagator can be written explicitly as the product $[4,19]$

$$
E=\left(I-I_{J} R_{J}^{T} I_{J}^{T} A\right) \cdots\left(I-I_{1} R_{1} I_{1}^{T} A\right) \cdots\left(I-I_{J} R_{J} I_{J}^{T} A\right) .
$$

Since the coarse problem is solved exactly, and since variational conditions hold, the coarse product term is an $A$-orthogonal projector

$$
I-I_{1} R_{1} I_{1}^{T} A=I-I_{1}\left(I_{1} A I_{1}^{T}\right)^{-1} I_{1}^{T} A=\left(I-I_{1}\left(I_{1} A I_{1}^{T}\right)^{-1} I_{1}^{T} A\right)^{2}=\left(I-I_{1} R_{1} I_{1}^{T} A\right)^{2} .
$$


Therefore, we may define $\bar{E}=\left(I-I_{1} R_{1} I_{1}^{T} A\right) \cdots\left(I-I_{J} R_{J} I_{J}^{T} A\right)$, and represent $E$ as the product $E=\bar{E}^{*} \bar{E}$. Now, since $A$ is $\mathrm{SPD}$, we have

$$
(A E v, v)=(A \bar{E} v, \bar{E} v) \geq 0 .
$$

Hence, $E$ is $A$-nonnegative. Under the conditions of the theorem, Lemma 3.1 implies that the preconditioner is SPD, and so by Lemma 2.9 it holds that $\|E\|_{A}<1$.

4. Additive Schwarz methods. We now present an analysis of additive Schwarz methods. We establish sufficient conditions for additive algorithms to yield SPD preconditioners. This theory is then employed to establish sufficient SPD conditions for additive DD and MG methods.

4.1. A sum operator. Consider a sum operator of the form

$$
E=I-B A=I-\omega\left(B_{0}+B_{1}\right) A, \quad \omega>0,
$$

where, as before, $A$ is an SPD operator, and $B_{0}$ and $B_{1}$ are linear operators on $\mathcal{H}$.

LEMMA 4.1. Sufficient conditions for symmetry and positivity of $B$, defined in (19), are as follows:

1. $B_{1}$ is $S P D$ in $\mathcal{H}$;

2. $B_{0}$ is symmetric and nonnegative on $\mathcal{H}$.

Proof. We have that $B=\omega\left(B_{0}+B_{1}\right)$, which is symmetric by the symmetry of $B_{0}$ and $B_{1}$. Positivity follows since $\left(B_{0} u, u\right) \geq 0$ and $\left(B_{1} u, u\right)>0 \forall u \in \mathcal{H}, u \neq 0$.

Remark 4.12. The parameter $\omega$ is usually required to make the additive method a convergent one. Its estimation is often nontrivial and can be very costly. As was noted in Remark 2.1, the parameter $\omega$ is not required when the linear additive method is used as a preconditioner in a conjugate gradients algorithm. This is exactly why additive MG and DD methods are used almost exclusively as preconditioners.

4.2. Additive DD. As in subsection 3.2, we consider the Hilbert space $\mathcal{H}$ and $J$ subspaces $I_{k} \mathcal{H}_{k}$ such that $I_{k} \mathcal{H}_{k} \subseteq \mathcal{H}=\sum_{k=1}^{J} I_{k} \mathcal{H}_{k}$. Again, we allow for the existence of a "coarse" subspace $I_{0} \mathcal{H}_{0} \subseteq \mathcal{H}$.

The error propagator of an additive DD method on the space $\mathcal{H}$ employing the subspaces $I_{k} \mathcal{H}_{k}$ has the general form (see [27])

$$
E=I-B A=I-\omega\left(I_{0} R_{0} I^{0}+I_{1} R_{1} I^{1}+\cdots+I_{J} R_{J} I^{J}\right) A .
$$

The operators $R_{k}$ are linear operators on $\mathcal{H}_{k}$, constructed in such a way that $R_{k} \approx$ $A_{k}^{-1}$, where the $A_{k}$ are the subdomain problem operators. Propagator (20) can be thought of as the sum method (19), by taking

$$
B_{0}=I_{0} R_{0} I^{0}, \quad B_{1}=\sum_{k=1}^{J} I_{k} R_{k} I^{k} .
$$

This identification allows for the use of Lemma 4.1 in order to establish conditions to guarantee that additive DD yields an SPD preconditioner. Before we state the main theorem, we need the following lemma, which characterizes the splitting of $\mathcal{H}$ into the subspaces $I_{k} \mathcal{H}_{k}$ in terms of a positive splitting constant $S_{0}$.

LEMma 4.2. Given any $v \in \mathcal{H}$, there exists a splitting $v=\sum_{k=1}^{J} I_{k} v_{k}, v_{k} \in \mathcal{H}_{k}$, and a constant $S_{0}>0$, such that

$$
\sum_{k=1}^{J}\left\|I_{k} v_{k}\right\|_{A}^{2} \leq S_{0}\|v\|_{A}^{2} .
$$


Proof. Since $\sum_{k=1}^{J} I_{k} \mathcal{H}_{k}=\mathcal{H}$, we can construct subspaces $\mathcal{V}_{k} \subseteq \mathcal{H}_{k}$, such that

$$
I_{k} \mathcal{V}_{k} \cap I_{l} \mathcal{V}_{l}=\{0\}, \text { for } k \neq l \text { and } \mathcal{H}=\sum_{k=1}^{J} I_{k} \mathcal{V}_{k}
$$

Any $v \in \mathcal{H}$ can be decomposed uniquely as $v=\sum_{k=1}^{J} I_{k} v_{k}, v_{k} \in \mathcal{V}_{k}$. Define the projectors $Q_{k} \in L\left(\mathcal{H}, I_{k} \mathcal{V}_{k}\right)$ such that $Q_{k} v=I_{k} v_{k}$. Then,

$$
\sum_{k=1}^{J}\left\|I_{k} v_{k}\right\|_{A}^{2}=\sum_{k=1}^{J}\left\|Q_{k} v\right\|_{A}^{2} \leq \sum_{k=1}^{J}\left\|Q_{k}\right\|_{A}^{2}\|v\|_{A}^{2} .
$$

Hence, the result follows with $S_{0}=\sum_{k=1}^{J}\left\|Q_{k}\right\|_{A}^{2}$.

THEOREM 4.3. Sufficient conditions for symmetry and positivity of the additive $D D$ operator $B$, defined in (20), are as follows:

1. $I^{k}=c_{k} I_{k}^{T}, \quad c_{k}>0, \quad k=0, \ldots, J$;

2. $R_{k}$ is $S P D$ on $\mathcal{H}_{k}, \quad k=1, \ldots, J$;

3. $R_{0}$ is symmetric and nonnegative on $\mathcal{H}_{0}$.

Proof. Symmetry of $B_{0}$ and $B_{1}$ follow trivially from the symmetry of $R_{k}$ and $R_{0}$ and from $I^{k}=c_{k} I_{k}^{T}$. That $B_{0}$ is nonnegative on $\mathcal{H}$ follows immediately from the nonnegativity of $R_{0}$ on $\mathcal{H}_{0}$.

Finally, we prove positivity of $B_{1}$. Define (only as a technical tool) the operators $A_{k}=I^{k} A I_{k}, k=1, \ldots, J$. By condition 1 , and the full rank nature of $I_{k}$, we have that $A_{k}$ is SPD. Now, since $R_{k}$ is also SPD, the product $R_{k} A_{k}$ is $A_{k}$-SPD. Hence, there exists an $\omega_{0}>0$ such that $0<\omega_{0}<\lambda_{i}\left(R_{k} A_{k}\right), k=1, \ldots, J$. This is used together with (21) to bound the sum

$$
\begin{gathered}
\sum_{k=1}^{J} c_{k}^{-1}\left(R_{k}^{-1} v_{k}, v_{k}\right)=\sum_{k=1}^{J} c_{k}^{-1}\left(A_{k} A_{k}^{-1} R_{k}^{-1} v_{k}, v_{k}\right) \\
\leq \sum_{k=1}^{J} c_{k}^{-1}\left(A_{k} v_{k}, v_{k}\right) \max _{v_{k} \neq 0} \frac{\left(A_{k} A_{k}^{-1} R_{k}^{-1} v_{k}, v_{k}\right)}{\left(A_{k} v_{k}, v_{k}\right)} \leq \sum_{k=1}^{J} c_{k}^{-1} \omega_{0}^{-1}\left(A_{k} v_{k}, v_{k}\right) \\
=\sum_{k=1}^{J} \omega_{0}^{-1}\left(A I_{k} v_{k}, I_{k} v_{k}\right)=\sum_{k=1}^{J} \omega_{0}^{-1}\left\|I_{k} v_{k}\right\|_{A}^{2} \leq\left(\frac{S_{0}}{\omega_{0}}\right)\|v\|_{A}^{2},
\end{gathered}
$$

with $v=\sum_{k=1}^{J} I_{k} v_{k}$. We can now employ this result to establish positivity of $B_{1}$. First note that

$$
\|v\|_{A}^{2}=(A v, v)=\sum_{k=1}^{J}\left(A v, I_{k} v_{k}\right)=\sum_{k=1}^{J}\left(I_{k}^{T} A v, v_{k}\right)=\sum_{k=1}^{J}\left(R_{k} c_{k}^{1 / 2} I_{k}^{T} A v, R_{k}^{-1} c_{k}^{-1 / 2} v_{k}\right) .
$$

By using the Cauchy-Schwarz inequality first in the $R_{k}$-inner-product and then in $\mathbb{R}^{J}$, we have that

$$
\begin{aligned}
\|v\|_{A}^{2} & \leq\left(\sum_{k=1}^{J}\left(R_{k} R_{k}^{-1} c_{k}^{-1 / 2} v_{k}, R_{k}^{-1} c_{k}^{-1 / 2} v_{k}\right)\right)^{1 / 2}\left(\sum_{k=1}^{J}\left(R_{k} c_{k}^{1 / 2} I_{k}^{T} A v, c_{k}^{1 / 2} I_{k}^{T} A v\right)\right)^{1 / 2} \\
& \leq\left(\frac{S_{0}}{\omega_{0}}\right)^{1 / 2}\|v\|_{A}\left(\sum_{k=1}^{J}\left(I_{k} R_{k} c_{k} I_{k}^{T} A v, A v\right)\right)^{1 / 2} \\
& =\left(\frac{S_{0}}{\omega_{0}}\right)^{1 / 2}\|v\|_{A}\left(B_{1} A v, A v\right)^{1 / 2} .
\end{aligned}
$$


Finally, division by $\|v\|_{A}$ and squaring yields

$$
\left(B_{1} A v, A v\right) \geq \frac{\omega_{0}}{S_{0}}\|v\|_{A}^{2}>0 \quad \forall v \in \mathcal{H}, \quad v \neq 0 .
$$

Remark 4.13. Condition 1 is naturally satisfied for $k=1, \ldots, J$, with $c_{k}=1$, since the associated $I_{k}$ and $I^{k}$ are usually inclusion and orthogonal projection operators (which are natural adjoints when the inner-products are inherited from the parent space, as in DD). The fact that $I^{0}=c_{0} I_{0}^{T}$ needs to be satisfied explicitly. Condition 2 requires the use of SPD subdomain solvers. The condition will hold, for example, when the subdomain solve is exact and the subdomain problem is SPD. (The latter is naturally satisfied by condition 1 and the full rank nature of $I_{k}$.) Finally, condition 3 is nontrivial and needs to be checked explicitly. The condition holds when the coarse space problem operator is SPD and the solve is exact. Note that variational conditions are not needed for the coarse space problem operator.

Consider again the case when the subspaces together do not span the entire space, except when the coarse space is included. The above theorem applies immediately with $R_{0}=0$, where now the coarse space is taken to be any one of the spaces $\mathcal{H}_{k}$, $k \neq 0$. The error propagator takes the form

$$
I-B A=I-\omega\left(I_{1} R_{1} I^{1}+I_{2} R_{2} I^{2}+\cdots+I_{J} R_{J} I^{J}\right) A .
$$

This leads to the following corollary.

COROLlARY 4.4. Sufficient conditions for symmetry and positivity of the additive $D D$ operator $B$, defined in (22), are as follows:

1. $I^{k}=c_{k} I_{k}^{T}, \quad c_{k}>0, \quad k=1, \ldots, J$;

2. $R_{k}$ is $S P D$ on $\mathcal{H}_{k}, \quad k=1, \ldots, J$.

4.3. Additive MG. As in subsection 3.3, given are the Hilbert space $\mathcal{H}$ and $J-1$ nested subspaces $I_{k} \mathcal{H}_{k}$ such that $I_{1} \mathcal{H}_{1} \subseteq I_{2} \mathcal{H}_{2} \subseteq \cdots \subseteq I_{J-1} \mathcal{H}_{J-1} \subseteq \mathcal{H}_{J} \equiv \mathcal{H}$. The operators $I_{k}$ and $I^{k}$ are the usual linear operators between the different spaces, as in the previous sections.

The error propagator of an additive MG method is defined explicitly:

$$
E=I-B A=I-\omega\left(I_{1} R_{1} I^{1}+I_{2} R_{2} I^{2}+\cdots+I_{J-1} R_{J-1} I^{J-1}+R_{J}\right) A .
$$

This can be thought of as the sum method analyzed earlier, by taking

$$
B_{0}=\sum_{k=1}^{J-1} I_{k} R_{k} I^{k}, \quad B_{1}=R_{J} .
$$

This identification allows for the use of Lemma 4.1 to establish sufficient conditions to guarantee that additive MG yields an SPD preconditioner.

THEOREM 4.5. Sufficient conditions for symmetry and positivity of the additive $M G$ operator $B$, defined in (23), are as follows:

1. $I^{k}=c_{k} I_{k}^{T}, \quad c_{k}>0, \quad k=1, \ldots, J-1$;

2. $R_{J}$ is $S P D$ in $\mathcal{H}$;

3. $R_{k}$ is symmetric nonnegative in $\mathcal{H}_{k}, \quad k=1, \ldots, J-1$.

Proof. Symmetry of $B_{0}$ and $B_{1}$ is obvious. $B_{1}$ is positive by condition 2. Nonnegativity of $B_{0}$ follows from

$$
\left(B_{0} u, u\right)=\sum_{k=1}^{J-1}\left(I_{k} R_{k}\left(c_{k} I_{k}\right)^{T} u, u\right)=\sum_{k=1}^{J-1} c_{k}\left(R_{k} I_{k}^{T} u, I_{k}^{T} u\right) \geq 0 \quad \forall u \in \mathcal{H}, u \neq 0 .
$$


Remark 4.14. Condition 1 of the theorem has to be imposed explicitly. Conditions 2 and 3 require the smoothers to be symmetric. The positivity of $R_{J}$ is satisfied when the fine grid smoother is convergent, although this is not a necessary condition. The nonnegativity of $R_{k}, k<J$, has to be checked explicitly. When the coarse problem operators are SPD, this condition is satisfied, for example, when the smoothers are nondivergent. Note that variational conditions for the subspace problem operators are not required.

Theorem 4.5 is applicable to the standard MG case, i.e., the case where the fine mesh smoother operates on the entire fine mesh. As in subsection 3.3, a different set of conditions is to be derived to cover the cases of (additive) hierarchical basis preconditioners [28] and additive multilevel methods with smoothing in local refinement regions only. The latter cases are treated most easily by loosening the restriction of nestedness of the spaces $I_{k} \mathcal{H}_{k}$. With $\mathcal{H}_{k}$ interpreted as the domain space of the smoother $R_{k}$, the theory becomes identical to that of the additive DD case. Sufficient conditions for the additive operator $B$ to be SPD are then similar to the conditions of the additive DD method in Corollary 4.4.

5. To symmetrize or not to symmetrize. The following lemma illustrates why symmetrizing is a bad idea for linear methods. It exposes the convergence rate penalty incurred by symmetrization of a linear method.

LEMma 5.1. For any $E \in \mathbf{L}(\mathcal{H}, \mathcal{H})$, it holds that

$$
\rho(E E) \leq\|E E\|_{A} \leq\|E\|_{A}^{2}=\left\|E E^{*}\right\|_{A}=\rho\left(E E^{*}\right) .
$$

Proof. The first and second inequalities hold for any norm. The first equality follows from Lemma 2.8, and the second follows from Lemma 2.1.

Note that this is an inequality not only for the spectral radii, which is only an asymptotical measure of convergence, but also for the $A$-norms of the nonsymmetric and symmetrized error propagators. The lemma illustrates that one may actually see the differing convergence rates early in the iteration as well.

Based on this lemma, and Corollary 2.14, we conjecture that when symmetrization of a linear method is required for its use as a preconditioner, the best results will be obtained by enforcing only a minimal amount of symmetry. This conjecture can be made more clear by considering the two linear methods with error propagators $E_{1}=E E$ and $E_{2}=E E^{*}$ and the effectiveness as preconditioners of the symmetrized product operators $E_{1} E_{1}^{*}$ and $E_{2} E_{2}^{*}$. Using Lemma 2.1 and Lemma 5.1, we find immediately that

$$
\left\|E_{1} E_{1}^{*}\right\|_{A}=\left\|E_{1}\right\|_{A}^{2} \leq\left\|E_{2}\right\|_{A}^{2}=\left\|E_{2} E_{2}^{*}\right\|_{A} .
$$

While both operators are symmetric, the operator $E_{1} E_{1}^{*}$ has been symmetrized "minimally" in the sense that the individual terms $E_{1}$ making up the product are themselves nonsymmetric. On the other hand, both of the terms $E_{2}$ making up the operator $E_{2} E_{2}^{*}$ are completely symmetric.

As a result of inequality (24) and Corollary 2.14, the bound for the condition number of the preconditioner associated with $E_{1} E_{1}^{*}$ is less than the corresponding bound for the preconditioner associated with $E_{2} E_{2}^{*}$. Hence, the "less symmetric" $E_{1} E_{1}^{*}$ would likely produce a better preconditioner than the "more symmetric" $E_{2} E_{2}^{*}$.

6. Numerical results. We present numerical results obtained by using multiplicative and additive finite-element-based DD and MG methods applied to two test problems, and we illustrate the theory of the preceding sections. 

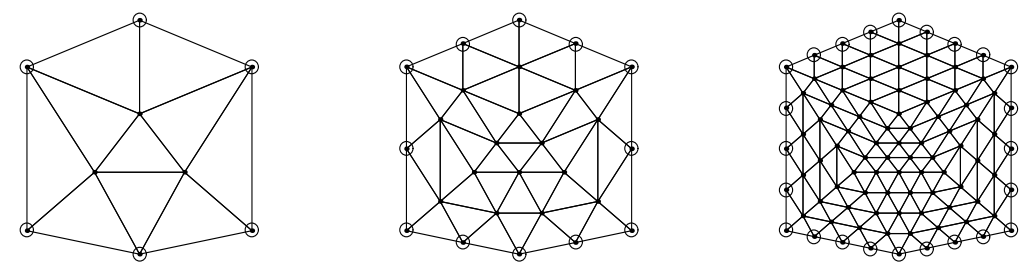

FIG. 1. Example 1: Nested finite element meshes for $M G$.
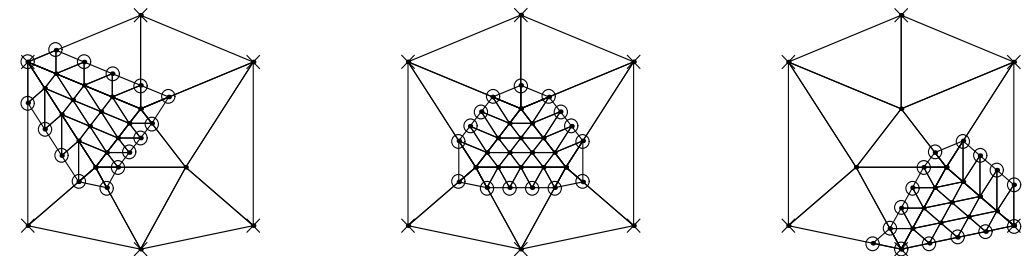

FIG. 2. Example 1: Overlapping subdomains for DD.

6.1. Example 1. Violation of variational conditions can occur in DD and MG methods when, for example, complex coefficient discontinuities do not lie along element boundaries on coarse meshes. An example of this occurs with the following test problem. The Poisson-Boltzmann equation describes the electrostatic potential of a biomolecule lying in an ionic solvent (see, e.g., [7] for an overview). This nonlinear elliptic equation for the dimensionless electrostatic potential $u(\mathbf{r})$ has the form

$$
-\nabla \cdot(\epsilon(\mathbf{r}) \nabla u(\mathbf{r}))+\bar{\kappa}^{2} \sinh (u(\mathbf{r}))=\left(\frac{4 \pi e_{c}^{2}}{k_{B} T}\right) \sum_{i=1}^{N_{m}} z_{i} \delta\left(\mathbf{r}-\mathbf{r}_{i}\right), \quad \mathbf{r} \in \mathbb{R}^{3}, \quad u(\infty)=0 .
$$

The coefficients appearing in the equation are discontinuous by several orders of magnitude. The placement and magnitude of atomic charges are represented by source terms involving delta functions. Analytical techniques are used to obtain boundary conditions on a finite domain boundary.

We will compare several MG and DD methods for a two-dimensional, linearized Poisson-Boltzmann problem, modeling a molecule with three point charges. The surface of the molecule is such that the discontinuities do not align with the coarsest mesh or with the subdomain boundaries. Beginning with the coarse mesh shown on the left in Figure 1, we uniformly refine the initial mesh of 10 elements (9 nodes) four times, leading to a fine mesh of 2560 elements (1329 nodes). Piecewise linear finite elements, combined with one point Gaussian quadrature, are used to discretize the problem. The three coarsest meshes used to formulate the MG methods are given in Figure 1. For the DD methods, the subdomains, corresponding to the initial coarse triangulation, are given a small overlap of one fine mesh triangle. The DD methods also employ a coarse space constructed from the initial triangulation. Figure 2 shows three overlapping subdomains overlaying the initial coarse mesh.

Computed results are presented in Tables 2 through 5. Given for each experiment is the number of iterations required to satisfy the error criterion (reduction of the $A$ norm of the error by $10^{-10}$ ). We report results for the unaccelerated, CG-accelerated, and Bi-CGstab-accelerated methods. Since the cost of one iteration differs for each method, Table 1 gives the operation counts per iteration, normalized by the cost of a single MG iteration. For the MG operation counts, two smoothing iterations 
TABLE 1

Normalized operation counts per iteration, Example 1.

\begin{tabular}{|c||c|c|c|}
\hline Method & UNACCEL & CG & Bi-CGstab \\
\hline \hline multiplicative MG & 1.0 & 1.4 & 2.6 \\
\hline additive MG & .95 & 1.3 & 2.5 \\
\hline multiplicative DD & 3.5 & 3.8 & 7.5 \\
\hline additive DD & 3.1 & 3.4 & 6.7 \\
\hline
\end{tabular}

by lexicographic Gauss-Seidel (one presmoothing and one postsmoothing) are used. The DD operation counts are for methods employing two sweeps through the subdomains, each approximate subdomain solve consisting of four sweeps of a Gauss-Seidel iteration.

Table 1 shows that multiplicative MG is slightly more costly than additive MG, since additive MG requires the computation of the residual only on the finest level. Similarly, multiplicative DD is somewhat more costly than additive DD, due to the need to update boundary information (recompute the residual) after the solution of each subdomain problem. Table 1 should not be used to compare MG and DD methods for efficiency. Similar experiments [15] with more carefully optimized DD and MG methods show DD to be often competitive with MG for difficult elliptic equations such as those with discontinuous coefficients, although there may be some debate as to which approach is more effective on parallel computers [24].

Multiplicative MG. The results for multiplicative V-cycle MG are presented in Table 2. Each row corresponds to a different smoothing strategy and is annotated by $\left(\nu_{1}, \nu_{2}\right)$, with $\nu_{1}$ indicating the presmoothing strategy, and $\nu_{2}$ the postsmoothing strategy. An "f" indicates the use of a single forward Gauss-Seidel sweep, while a "b" denotes the use of the adjoint of the latter, i.e., a backward Gauss-Seidel sweep. $\left(\nu_{1}, \nu_{2}\right)=(f f, f b)$, for example, corresponds to two forward Gauss-Seidel presmoothing steps and a symmetric (forward/backward) postsmoothing step. Two series of results are given. For the first set, we explicitly imposed the Galerkin conditions when constructing the coarse operators. In this case, the multigrid algorithm is guaranteed to converge by Theorem 3.8. In the second series of tests, corresponding to the numbers in parentheses, the coarse mesh operators are constructed using standard finite element discretization. In that case, Galerkin conditions are not satisfied everywhere due to coefficient discontinuities appearing within coarse elements; hence, the MG method may diverge (DIV).

The unaccelerated MG results clearly illustrate the symmetry penalty discussed in section 5. The nonsymmetric methods are always superior to the symmetric ones (the cases $(\mathrm{f}, \mathrm{b}),(\mathrm{ff}, \mathrm{bb})$, and $(\mathrm{fb}, \mathrm{fb}))$. Note that minimal symmetry (ff,bb) leads to a better convergence than maximal symmetry (fb,fb). The correctness of Lemma 5.1 is illustrated by noting that two iterations of the (f,0) strategy are actually faster than one iteration of the (f,b) strategy; also, compare the (ff,0) strategy with the (ff,bb) one. CG-acceleration leads to a guaranteed reduction in iteration count for the symmetric preconditioners (see Lemma 2.15). We observe that the unaccelerated method need not be convergent for CG to be effective (recall Remarks 2.1 and 4.12, and the (f,b) result). CG appears to accelerate some nonsymmetric linear methods also. Yet, it seems difficult to predict failure or success beforehand in such cases. The most robust method appears to be the Bi-CGstab method. The number of iterations with this method depends only marginally on the symmetric or nonsymmetric nature of the linear method. Note the tendency to favor the nonsymmetric V-cycle strate- 
TABLE 2

Example 1: Multiplicative MG with variational (discretized) coarse problem.

\begin{tabular}{|rl||rl|rl|rl|}
\hline \multicolumn{1}{|c||}{$\nu_{1}$} & $\nu_{2}$ & \multicolumn{2}{|c|}{ UNACCEL } & \multicolumn{2}{|c|}{ CG } & \multicolumn{2}{|c|}{ Bi-CGstab } \\
\hline \hline $\mathrm{f}$ & 0 & 65 & $(\mathrm{DIV})$ & $\gg 100$ & $(\gg 100)$ & 14 & $(16)$ \\
\hline \hline $\mathrm{f}$ & $\mathrm{b}$ & 55 & $(\mathrm{DIV})$ & 16 & $(18)$ & 10 & $(15)$ \\
\hline $\mathrm{f}$ & $\mathrm{f}$ & 40 & $(31)$ & 30 & $(\gg 100)$ & 9 & $(9)$ \\
\hline $\mathrm{ff}$ & 0 & 39 & $(48)$ & $\gg 100$ & $(\gg 100)$ & 8 & $(10)$ \\
\hline $\mathrm{fb}$ & 0 & 53 & $(\mathrm{DIV})$ & $\gg 100$ & $(\gg 100)$ & 10 & $(11)$ \\
\hline 0 & $\mathrm{ff}$ & 39 & $(29)$ & 29 & $(\gg 100)$ & 8 & $(9)$ \\
\hline 0 & $\mathrm{fb}$ & 53 & $(\mathrm{DIV})$ & 17 & $(99)$ & 10 & $(12)$ \\
\hline \hline $\mathrm{fb}$ & $\mathrm{fb}$ & 34 & $(27)$ & 12 & $(13)$ & 8 & $(8)$ \\
\hline $\mathrm{ff}$ & $\mathrm{bb}$ & 28 & $(18)$ & 11 & $(11)$ & 7 & $(7)$ \\
\hline $\mathrm{ff}$ & $\mathrm{ff}$ & 24 & $(15)$ & 12 & $(12)$ & 6 & $(6)$ \\
\hline $\mathrm{fff}$ & $\mathrm{f}$ & 24 & $(15)$ & 17 & $(27)$ & 6 & $(6)$ \\
\hline $\mathrm{ffff}$ & 0 & 25 & $(17)$ & $\gg 100$ & $(\gg 100)$ & 7 & $(6)$ \\
\hline
\end{tabular}

TABLE 3

Example 1: Multiplicative DD with variational (discretized) coarse problem.

\begin{tabular}{|c|c|c|c|c|c|c|c|}
\hline Accel. & subdomain solve & \multicolumn{2}{|c|}{ forw } & \multicolumn{2}{|c|}{ forw/back } & \multicolumn{2}{|c|}{ forw/forw } \\
\hline \multirow[t]{4}{*}{ UNACCEL } & exact & $\overline{40}$ & $\overline{(42)}$ & $\overline{38}$ & $\overline{(39)}$ & $\overline{20}$ & $\overline{(21)}$ \\
\hline & symmetric & 279 & $(282)$ & 146 & $(149)$ & 140 & (141) \\
\hline & adjointed & - & & 110 & $(112)$ & 102 & $(103)$ \\
\hline & nonsymmetric & 189 & (191) & 102 & (104) & 95 & $(96)$ \\
\hline \multirow[t]{4}{*}{$\mathrm{CG}$} & exact & $\gg 500$ & $(\gg 500)$ & 13 & $(13)$ & 20 & $(20)$ \\
\hline & symmetric & 140 & $(56)$ & 24 & (24) & 29 & $(27)$ \\
\hline & adjointed & - & - & 21 & $(21)$ & 25 & $(26)$ \\
\hline & nonsymmetric & 135 & $(83)$ & 22 & $(23)$ & 28 & $(28)$ \\
\hline \multirow[t]{4}{*}{ Bi-CGstab } & exact & 9 & (9) & 9 & (9) & 6 & (6) \\
\hline & symmetric & 23 & $(23)$ & 17 & $(16)$ & 16 & $(16)$ \\
\hline & adjointed & - & - & 14 & $(14)$ & 14 & $(13)$ \\
\hline & nonsymmetric & 19 & $(20)$ & 13 & (13) & 13 & (13) \\
\hline
\end{tabular}

gies. Overall, the fastest method proves to be the Bi-CGstab-acceleration of a (very nonsymmetric) $\mathrm{V}(1,0)$-cycle.

Multiplicative DD. Some numerical results for multiplicative DD with different subdomain solvers, and different subdomain sweeps are given in Table 3. In the column "forw," the iteration counts reported were obtained with a single sweep though the subdomains on each multiplicative DD iteration. The other columns correspond to a symmetric forward/backward sweep or to two forward sweeps. Four different subdomain solvers are used: an exact solve, a symmetric method consisting of two symmetric Gauss-Seidel iterations, a nonsymmetric method consisting of four GaussSeidel iterations, and, finally, a method using four forward Gauss-Seidel iterations in the forward subdomain sweep and using their adjoint, i.e., four backward GaussSeidel iterations, in the backward subdomain sweep. The latter leads to a symmetric iteration; see Remark 3.3. Note that the cost of the three inexact subdomain solvers is identical.

Although apparently not as sensitive to operator symmetries as MG, the same conclusions can be drawn for DD as for MG. In particular, the symmetry penalty is seen for the pure DD results. Lemma 5.1 is confirmed since two iterations in the column "forw" are always more efficient than one iteration of the corresponding symmetrized method in column "forw/back." The CG results indicate that using minimal symmetry (the "adjointed" column) is a more effective approach than the 
TABLE 4

Example 1: Additive $M G$ with variational (discretized) coarse problem.

\begin{tabular}{|c||rc|rl|ll|}
\hline \multicolumn{1}{|c||}{} & \multicolumn{2}{|c|}{ UNACCEL } & \multicolumn{2}{|c|}{ CG } & \multicolumn{2}{|c|}{ Bi-CGstab } \\
\hline \hline $\mathrm{f}$ & 175 & $(\gg 1000)$ & $\gg 100$ & $(\gg 100)$ & 23 & $(52)$ \\
\hline \hline $\mathrm{ff}$ & 110 & $(\gg 1000)$ & 119 & $(168)$ & 19 & $(43)$ \\
\hline $\mathrm{fb}$ & 146 & $(\gg 1000)$ & 34 & $(54)$ & 23 & $(49)$ \\
\hline \hline $\mathrm{ffff}$ & 95 & $(\gg 1000)$ & 28 & $(67)$ & 17 & $(37)$ \\
\hline $\mathrm{ffbb}$ & 100 & $(\gg 1000)$ & 27 & $(47)$ & 17 & $(34)$ \\
\hline $\mathrm{fbfb}$ & 95 & $(\gg 1000)$ & 28 & $(48)$ & 20 & $(43)$ \\
\hline
\end{tabular}

TABLE 5

Example 1: Additive DD with variational (discretized) coarse problem.

\begin{tabular}{|c||cc|cc|cc|}
\hline \multicolumn{1}{|c||}{ subdomain solve } & \multicolumn{2}{|c|}{ UNACCEL } & \multicolumn{2}{c|}{ CG } & \multicolumn{2}{c|}{ Bi-CGstab } \\
\hline \hline exact & $\gg 1000$ & $(\gg 1000)$ & 34 & $(34)$ & 25 & $(27)$ \\
\hline symmetric & $\gg 1000$ & $(\gg 1000)$ & 57 & $(57)$ & 50 & $(49)$ \\
\hline nonsymmetric & $\gg 1000$ & $(\gg 1000)$ & 69 & $(65)$ & 38 & $(41)$ \\
\hline
\end{tabular}

fully symmetric one (the "symmetric" column). Again, the most robust acceleration is the Bi-CGstab one.

Additive MG. Results obtained with an additive MG method are reported in Table 4 . The number and nature of the smoothing strategy is given in the first column of the table.

In the case of an unaccelerated additive method, the selection of a good damping parameter is crucial for convergence of the method. We did not search extensively for an optimal parameter; a selection of $\omega=0.45$ seemed to provide good results in the case when the coarse problem is variationally defined. No $\omega$-value leading to satisfactory convergence was found in the case when the coarse problem is obtained by discretization. In the case of CG acceleration the observed convergence behavior was completely independent of the choice of $\omega$; see Remark 3.3. The symmetric methods $(\nu=f b, f f b b, f b f b)$ are accelerated very well. Some of the nonsymmetric methods are accelerated too, especially when the number of smoothing steps is sufficiently large. In the case of Bi-CGstab-acceleration, there appeared to be a dependence of convergence on $\omega$ (only with use of nonvariational coarse problem). In that case we took $\omega=1$. The overall best method appears to be the Bi-CGstab acceleration of the nonsymmetric MG method with a single forward Gauss-Seidel sweep on each grid level.

Additive DD. The results for additive DD are given in Table 5. The subdomain solver is either an exact solver, a symmetric solver based on two symmetric (forward/backward) Gauss-Seidel sweeps, or a nonsymmetric solver based on four forward Gauss-Seidel iterations.

No value of $\omega$ was found that led to satisfactory convergence of the unaccelerated method. CG-acceleration performs well when the linear method is symmetric; it performs less well for the nonsymmetric method.

6.2. Example 2. The second test problem is the Laplace equation on a semiadapted L-shaped domain, with Dirichlet boundary conditions chosen in such a way that the equation has the following solution (in polar coordinates):

$$
u(r, \theta)=\sqrt{r} \sin (\theta / 2),
$$



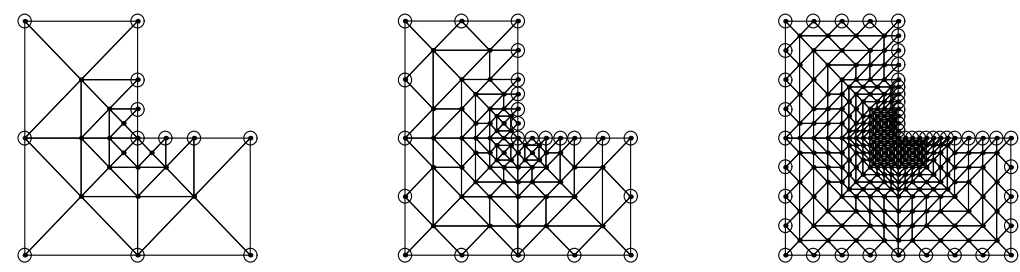

FIG. 3. Example 2: Nested finite element meshes for MG.
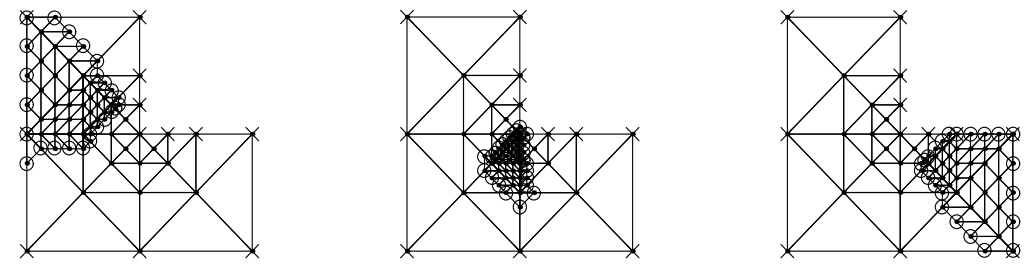

FIG. 4. Example 2: Overlapping subdomains for DD.

TABLE 6

Example 2: Multiplicative $M G$.

\begin{tabular}{|cc||c|c|c|}
\hline$\nu_{1}$ & $\nu_{2}$ & UNACCEL & CG & Bi-CGstab \\
\hline \hline $\mathrm{f}$ & 0 & 33 & $\gg 100$ & 11 \\
\hline \hline $\mathrm{f}$ & $\mathrm{b}$ & 23 & 12 & 7 \\
\hline $\mathrm{f}$ & $\mathrm{f}$ & 19 & 22 & 6 \\
\hline $\mathrm{ff}$ & 0 & 21 & $\gg 100$ & 7 \\
\hline $\mathrm{fb}$ & 0 & 25 & $\gg 100$ & 8 \\
\hline 0 & $\mathrm{ff}$ & 20 & 42 & 7 \\
\hline 0 & $\mathrm{fb}$ & 23 & 17 & 8 \\
\hline \hline $\mathrm{fb}$ & $\mathrm{fb}$ & 16 & 9 & 6 \\
\hline $\mathrm{ff}$ & $\mathrm{bb}$ & 15 & 9 & 5 \\
\hline $\mathrm{ff}$ & $\mathrm{ff}$ & 14 & 9 & 5 \\
\hline $\mathrm{fff}$ & $\mathrm{f}$ & 14 & 12 & 5 \\
\hline $\mathrm{ffff}$ & 0 & 16 & 36 & 5 \\
\hline
\end{tabular}

where the re-entrant corner in the domain is located at the origin. Note that the one point Gaussian quadrature rule which we employ to construct the stiffness matrix entries is an exact integrator here. Hence, the variational conditions (12) hold automatically between the fine space and all subdomain and coarse spaces for both the MG and the DD methods.

Figure 3 shows a nested sequence of uniform mesh refinements used to formulate the MG methods. (A total of five mesh levels is used in the computation.) Figure 4 shows several overlapping subdomains constructed from a piece of the fine mesh of 9216 elements (4705 nodes) overlaying the initial coarse mesh of 36 elements (25 nodes).

Multiplicative methods. The results for multiplicative MG are given in Table 6, whereas the results for multiplicative DD are given in Table 7 . The results are similar to those for Example 1; in particular, imposing minimal symmetry is the most effective CG-accelerated approach to the problem. Employing the least symmetric linear method alone is the most effective linear method, and the same nonsymmetric linear method yields the most effective Bi-CGstab-accelerated approach. 
TABLE 7

Example 2: Multiplicative DD.

\begin{tabular}{|c|c|c|c|c|}
\hline Accel. & subdomain solve & forw & forw/back & forw/forw \\
\hline \hline UNACCEL & exact & 73 & 60 & 37 \\
\cline { 2 - 5 } & symmetric & 402 & 205 & 207 \\
\cline { 2 - 5 } & adjointed & - & 153 & 146 \\
\cline { 2 - 5 } & nonsymmetric & 267 & 144 & 134 \\
\hline \multirow{4}{*}{ CG } & exact & 116 & 17 & 17 \\
\cline { 2 - 5 } & symmetric & 164 & 37 & 38 \\
\cline { 2 - 5 } & adjointed & - & 32 & 33 \\
\cline { 2 - 5 } & nonsymmetric & 121 & 31 & 32 \\
\hline Bi-CGstab & exact & 11 & 11 & 7 \\
\cline { 2 - 5 } & symmetric & 37 & 25 & 26 \\
\cline { 2 - 5 } & adjointed & - & 22 & 21 \\
\cline { 2 - 5 } & nonsymmetric & 27 & 21 & 23 \\
\hline
\end{tabular}

TABLE 8

Example 2: Additive $M G$.

\begin{tabular}{|c||c|c|c|}
\hline$\nu$ & UNACCEL & CG & Bi-CGstab \\
\hline \hline $\mathrm{f}$ & 91 & $\gg 1000$ & 21 \\
\hline \hline $\mathrm{ff}$ & 62 & 31 & 16 \\
\hline $\mathrm{fb}$ & 74 & 29 & 18 \\
\hline \hline $\mathrm{ffff}$ & 126 & 25 & 14 \\
\hline $\mathrm{ffbb}$ & 136 & 27 & 15 \\
\hline $\mathrm{fbfb}$ & 98 & 27 & 15 \\
\hline
\end{tabular}

TABLE 9

Example 2: Additive DD.

\begin{tabular}{|c||c|c|c|}
\hline subdomain solve & UNACCEL & CG & Bi-CGstab \\
\hline \hline exact & $\gg 1000$ & 42 & 29 \\
\hline symmetric & $\gg 1000$ & 86 & 56 \\
\hline nonsymmetric & $\gg 1000$ & 82 & 49 \\
\hline
\end{tabular}

Additive methods. As for Example 1, in the case of the unaccelerated additive methods the selection of the damping parameter was crucial for convergence of the methods. We did not search extensively for an optimal parameter; a selection of $\omega=0.45$ seemed to provide acceptable results for DD. Note that improved convergence behavior might be obtained by allowing different $\omega$ values for each subdomain solver (this will not be further investigated here). No satisfactory value for $\omega$ was found for additive MG. In the case of CG acceleration, the observed convergence behavior was completely independent of the choice of $\omega$. The results for additive MG are given in Table 8, whereas the results for additive DD are given in Table 9. The effect of the symmetry of the linear method's error propagator on its convergence, and on the convergence behavior of CG and Bi-CGstab, was as for Example 1.

7. Concluding remarks. In this paper, we developed framework for establishing sufficient conditions which guarantee that abstract multiplicative and additive Schwarz algorithms yield self-adjoint positive definite preconditioners. We then analyzed four specific methods-MG and DD methods, in both their additive and multiplicative forms. In all four cases, we used the general theory to establish sufficient conditions that guarantee the resulting preconditioner is SPD. As discussed in Remarks $3.3,3.8,4.13$, and 4.14 , the sufficient conditions for the theory, in the case of all 
four methods, are easily satisfied for nonvariational and even nonconvergent methods. The analysis shows that by simply taking some care in the way a Schwarz method is formulated, one can guarantee that the method is convergent when accelerated with the conjugate gradient method.

We also investigated the role of symmetry in linear methods and preconditioners. A certain penalty lemma (Lemma 5.1) was stated and proved, illustrating why symmetrizing is actually a bad idea for linear methods. It was conjectured that enforcing minimal symmetry in a linear preconditioner achieves the best results when combined with the conjugate gradient method, and our numerical examples illustrate this behavior almost uniformly. A sequence of experiments with two nontrivial test problems showed that the most efficient approach may be to abandon symmetry in the preconditioner altogether and to employ a nonsymmetric solver such as Bi-CGstab. While acceleration with CG was strongly dependent on the symmetric nature of the preconditioner, Bi-CGstab always converged rapidly. In addition, BiCGstab appeared to benefit from the behavior predicted by Lemma 5.1, namely, that a nonsymmetric linear preconditioner should have better convergence properties than its symmetrized form.

Acknowledgments. The authors thank the referees and Olof Widlund for several helpful comments.

\section{REFERENCES}

[1] S. Ashby, M. Holst, T. Manteuffel, and P. Saylor, The Role of the Inner Product in Stopping Criteria for Conjugate Gradient Iterations, Tech. report UCRL-JC-112586, Lawrence Livermore National Laboratory, Livermore, CA, 1992.

[2] S. F. Ashby, T. A. Manteuffel, And P. E. Saylor, A taxonomy for conjugate gradient methods, SIAM J. Numer. Anal., 27 (1990), pp. 1542-1568.

[3] R. E. Bank, T. F. Dupont, And H. Yserentant, The hierarchical basis multigrid method, Numer. Math., 52 (1988), pp. 427-458.

[4] J. H. Bramble, J. E. Pasciak, J. Wang, and J. Xu, Convergence estimates for multigrid algorithms without regularity assumptions, Math. Comp., 57 (1991), pp. 23-45.

[5] J. H. Bramble, J. E. Pasciak, J. Wang, and J. Xu, Convergence estimates for product iterative methods with applications to domain decomposition and multigrid, Math. Comp., 57 (1991), pp. 1-21.

[6] A. Brandt, Multi-level adaptive solutions to boundary-value problems, Math. Comp., 31 (1977), pp. 333-390.

[7] J. M. Briggs And J. A. MCCAmmon, Computation unravels mysteries of molecular biophysics, Comput. in Phy., 6 (1990), pp. 238-243.

[8] P. Concus, G. H. Golub, AND D. P. O'LeAry, A generalized conjugate gradient method for the numerical solution of elliptic partial differential equations, in Sparse Matrix Computations, J. R. Bunch and D. J. Rose, eds., Academic Press, New York, 1976, pp. 309-332.

[9] M. DRYja AND O. B. Widlund, Towards a unified theory of domain decomposition algorithms for elliptic problems, in Third International Symposium on Domain Decomposition Methods for Partial Differential Equations, T. F. Chan, R. Glowinski, J. Périaux, and O. B. Widlund, eds., SIAM, Philadelphia, PA, 1989, pp. 3-21.

[10] R. P. Fedorenko, A relaxation method for solving elliptic difference equations, USSR Comput. Math. and Math. Phys., 1(5) (1961), pp. 1092-1096.

[11] W. HackBusch, Multi-grid Methods and Applications, Springer-Verlag, Berlin, 1985.

[12] W. Hackbusch, Iterative Solution of Large Sparse Systems of Equations, Springer-Verlag, Berlin, 1994.

[13] M. R. Hestenes And E. Stiefel, Methods of conjugate gradients for solving linear systems, J. Research of NBS, 49 (1952), pp. 409-435.

[14] M. Holst, An Algebraic Schwarz Theory, Tech. report CRPC-94-12, Applied Mathematics and CRPC, California Institute of Technology, Pasadena, CA, 1994.

[15] M. Holst AND F. SAIED, Multigrid and domain decomposition methods for electrostatics problems, in Domain Decomposition Methods in Science and Engineering, D. E. Keyes and J. Xu, eds., American Mathematical Society, Providence, RI, 1995. 
[16] D. E. Keyes And J. Xu, EdS., Domain Decomposition Methods in Science and Engineering, American Mathematical Society, Providence, RI, 1995.

[17] R. Kress, Linear Integral Equations, Springer-Verlag, Berlin, 1989.

[18] E. Kreyszig, Introductory Functional Analysis with Applications, John Wiley, New York, 1990.

[19] S. F. MCCormick ANd J. W. Ruge, Unigrid for multigrid simulation, Math. Comp., 41 (1983), pp. 43-62.

[20] J. M. OrTega, Numerical Analysis: A Second Course, Academic Press, New York, 1972.

[21] Y. SaAd And M. H. Schultz, GMRES: A generalized minimal residual algorithm for solving nonsymmetric linear systems, SIAM J. Sci. Statist. Comput., 7 (1986), pp. 856-869.

[22] H. A. Schwarz, Über einige Abbildungsaufgaben, Ges. Math. Abh., 11 (1869), pp. 65-83.

[23] P. Sonneveld, CGS: A fast Lanczos-type solver for nonsymmetric linear systems, SIAM J. Sci. Statist. Comput., 10 (1989), pp. 36-52.

[24] E. F. VAN DE Velde, Domain Decomposition vs. Concurrent Multigrid, Tech. report CRPC94-11, Applied Mathematics and CRPC, California Institute of Technology, Pasadena, CA, 1994.

[25] H. A. VAN DER VORST, BI-CGSTAB: A fast and smoothly converging variant of BI-CG for the solution of nonsymmetric linear systems, SIAM J. Sci. Statist. Comput., 13 (1992), pp. 631-644.

[26] J. Xu, Theory of Multilevel Methods, Ph.D. thesis, Department of Mathematics, Penn State University, University Park, PA, 1989; Tech. report AM 48.

[27] J. Xu, Iterative methods by space decomposition and subspace correction, SIAM Rev., 34 (1992), pp. 581-613.

[28] H. Yserentant, On the multi-level splitting of finite element spaces, Numer. Math., 49 (1986), pp. 379-412. 